Revue d'histoire de l'Amérique française

REVUE D.HISTOIRE DE L'AMÉRIQUE FRANÇAISE

\title{
Le commerce de détail au Canada (1870-1880)
}

\section{Gaétan Gervais}

Volume 33, numéro 4, mars 1980

URI : https://id.erudit.org/iderudit/303811ar

DOI : https://doi.org/10.7202/303811ar

Aller au sommaire du numéro

Éditeur(s)

Institut d'histoire de l'Amérique française

ISSN

0035-2357 (imprimé)

1492-1383 (numérique)

Découvrir la revue

Citer cet article

Gervais, G. (1980). Le commerce de détail au Canada (1870-1880). Revue

d'histoire de l'Amérique française, 33(4), 521-556.

https://doi.org/10.7202/303811ar d'utilisation que vous pouvez consulter en ligne.

https://apropos.erudit.org/fr/usagers/politique-dutilisation/ 


\title{
LE COMMERCE DE DÉTAIL AU CANADA (1870-1880)*
}

\author{
GAÉTAN GERVAIS \\ Université Laurentienne \\ Sudbury
}

La spécialisation grandissante qui accompagne le développement du capitalisme occidental au XIXe siècle fait croître, entre les producteurs et les consommateurs, une foule d'intermédiaires dont le rôle consiste à assurer l'échange des marchandises, soit entre les régions, soit entre la ville et la campagne. Les produits circulent grâce à cette armée d'importateurs-exportateurs, de grossistes, d'expéditeurs, d'agents et, au bas de la pyramide, de détaillants. Bénéficiaires et souvent promoteurs de l'expansion des transports maritimes et terrestres, ces intermédiaires forment un réseau complexe de distributeurs dont les chaînons inférieurs sont les petits commerçants et les détaillants de tout genre. C'est à ce dernier niveau que les consommateurs achètent les produits.

Si le développement des transports, que les historiens canadiens ont peut-être trop associé à une volonté politique, a suscité un assez grand intérêt, l'évolution du système de distribution des marchandises, notamment au niveau du commerce de détail, a peu retenu leur attention ${ }^{1}$. En effet, il n'existe pas encore d'histoire du

* L'auteur tient à remercier ses collègues qui ont lu ce texte et fait des suggestions utiles: MM. Jean Roy et Angus Gilbert, ainsi que Mme Enid Barnett.

1 Les études d'histoire économique du Canada se sont peu penchées sur le petit commerce: voir par exemple W.T. Easterbrook et H. J. Aitken, Canadian Economic History (Toronto, Macmillan, 1968), xiii-606p; O.F. McDiarmid, Commercial Policy in the Canadian Economy (Cambridge, Mass. Harvard University Press, 1946), xii-397p.; O.D. Skelton, "General Economic History of the Dominion 1867-1912", dans Canada and Its Provinces (Toronto, Publishers' Association of Canada, 1913), V: 95-274. Il existe cependant une exception, car dans leur Histoire économique du Québec 1851-1896 (Montréal, Fides, XXXVIII-436p.), Jean Hamelin et Yves Roby consacrent la cinquième partie de leur ouvrage aux “échanges” (p. 325-365) et décrivent les rouages du petit commerce. C'est à notre connaissance la seule présentation systématique du réseau de distribution canadien des marchandises, et le seul endroit où le problème des échanges et du commerce est posé en termes de réseau de distribution. On trouvera aussi quelques monographies sur des maisons d'affaires ou des commerçants, et quelques articles anecdotiques tels que D. Armstrong, "Merchandising on the frontier", dans Canadian Magazine (1913) XV: 377-385. Aux Etats-Unis, la question a été mieux étudiée par L.A. Johnson, Over the Counter and on the shelf. Country storekeeping in America, 1620-1920 (Rutland, Vt, Charles Tuttle Company, c. 1961 , xii-140p.), ouvrage qui, malgré son caractère parfois anecdotique, contient beaucoup de renseignements utiles. 
commerce au Canada, lacune que peut expliquer l'absence de fonds d'archives importants sur cette question.

Vers la fin du XIXe siècle, commerce de détail est à peu près synonyme de petit commerce: le monde occidental ne connaît pas encore, dans la vente, la mutation que Peter Mathias appelle la "retailing revolution"2. Celle-ci donne naissance, au XXe siècle, surtout dans les villes, à de vastes magasins de vente au détail, parfois organisés en chaînes. Au Canada, le premier exemple de ce type de grand magasin est sans doute celui que Timothy Eaton ouvre à Toronto en $1869^{3}$. Mais vers 1870 , il faut entendre par "détaillant" ou "petit commerce", cette longue liste de marchands généraux éparpillés dans les villages et dans les quartiers des villes. Bien que l'on retrouve parfois dans les centres urbains, plus rarement à la campagne, des boutiques spécialisées (des tailleurs, des cordonniers, des boulangers, etc.), les marchands font généralement le commerce d'une grande variété de produits manufacturés (les "dry goods") et de produits alimentaires (les "groceries"). Ce commerçant général possédant un magasin de petite taille est typique des marchands de l'époque; il constitue le rouage essentiel d'un réseau de distribution des marchandises dans une économie de plus en plus monétaire et spécialisée ${ }^{4}$.

Ainsi, ces petits commerces sont appelés à jouer un double rôle: premièrement distribuer aux consommateurs les produits manufacturés ou alimentaires, et deuxièmement constituer une source de crédit pour leurs clients. La connaissance intime de leurs milieux et de leurs clients permet aux marchands généraux de se rendre indispensables. Ils deviennent facilement des créanciers, voire des spéculateurs sur les produits ou sur les biens fonciers. Durant la décennie 1870-1880, deux facteurs jouent considérablement sur l'évolution de ce secteur économique: le développement des transports et la crise économique qui sévit à partir de 1874 , le premier comme facteur d'expansion, le second comme facteur de ralentissement de l'activité commerciale. Ces pressions contradictoires ont pour conséquence d'éliminer un grand nombre de petites entreprises, assainissement que d'aucuns jugent opportun.

2 Peter Mathias, The Retailing Revolution, 1967.

3 William Stephenson, The Store that Timothy Built, Toronto, 1969.

4 C'est aussi la conclusion que tirent Hamelin et Roby: “Le commerce de détail n'est pas encore spécialisé dans les villes. Les branches les plus importantes sont les épiceries, les quincailleries, les librairies et les 'druggistes'. Il existe avant tout des magasins généraux où l'on offre de tout." Hamelin et Roby, Histoire économique..., 345. 
Pour étudier le commerce de détail canadien, nous disposons, à partir de 1867 , d'une source intéressante mais peu exploitée jusqu'ici: le Monetary Times. Cet hebdomadaire permet de saisir, pour les fins de cet article, les grandes caractéristiques du petit commerce, son organisation, son fonctionnement, ses difficultés et, surtout, tout le système de crédit qui le soutient durant les années soixante-dix du XIXe siècle, années marquées d'une profonde crise économique qui fait mieux ressortir certaines faiblesses de ce commerce de détail. Le Monetary Times se voue aux intérêts des petits commerçants, mais il aborde aussi tous les autres aspects de la vie économique canadienne. Dans ce journal, qui devient ainsi une mine de renseignements sur l'économie canadienne, le lecteur trouve des articles sur les divers secteurs manufacturiers, sur la conjoncture économique, sur les sociétés commerciales, sur les techniques de gestion des entreprises. Les rédacteurs prodiguent à chaque occasion des conseils aux petits commerçants. Cette publication constitue donc une excellente source pouvant faire connaître l'état et les difficultés du petit commerce, mais aussi les opinions et l'idéologie des marchands durant cette période. Le journal circule "most largely among shop-keepers and tradespeople in Canada"s. Il paraît hebdomadairement à Toronto et semble jouer au Canada le rôle que tiennent aux États-Unis des revues ou journaux tels que le American Grocer, le Country Merchant de SanFrancisco, le Dry Goods Journal de Philadelphie et le Journal of Commerce de Saint-Louis.

La première partie de cet article examine les facteurs d'expansion dans le commerce de détail vers 1870; la deuxième section analyse la situation du commerce de détail ainsi que les éléments qui soutiennent cette activité. Enfin, dans une dernière partie, les effets de la crise économique sur le commerce de détail sont analysés.

\section{I- LES FACTEURS D'EXPANSION DANS LE COMMERCE}

La conjoncture, vers 1870, favorise de deux façons les marchands: premièrement, en se trouvant dans un pays nouveau où l'accumulation des biens est faible, ils profitent de la demande que génère inévitablement un tel milieu. Deuxièmement, et c'est notamment le cas dans les années précédant 1873 , il existe une pression pour vendre venant de l'expansion des manufactures dans les pays capitalistes industrialisés.

$5 \mathrm{M}$ [onetary] T[imes], 11 décembre 1885, 661. 
Dans l'évolution économique du XIXe siècle, les années soixante-dix se situent au sommet du cycle kondratieff dit des chemins de fer (1847-1897) : ce cycle séculaire atteint son point culminant en 1873 puis sombre dans la "grande dépression" de la fin $\mathrm{du}$ siècle. Nous avons donc dans la décennie soixante-dix trois moments: les années 1870 à 1873 se caractérisent par la grande prospérité de la fin de la phase A du kondratieff; entre 1874 et 1878, une profonde crise ébranle les économies occidentales; enfin, l'année 1878 marque le début d'une légère reprise économique et d'un modeste redressement coïncidant au Canada avec l'introduction de la "politique nationale" du parti conservateur6.

Les historiens canadiens ont traditionnellement perçu cette politique comme une importante mesure réalisant dans l'ordre économique le programme confédératif de 1864-1867. D'autre part, ce programme économique peut aussi traduire un changement fondamental par lequel l'économie canadienne passe d'un stade où domine le capitalisme commercial (et les projets chimériques de la bourgeoisie commerciale du Saint-Laurent qui rêve de capturer le commerce céréalier de l'Ouest américain), à une nouvelle phase de développement où s'affirme un capitalisme de type industriel ou manufacturier, correspondant à la montée d'une nouvelle classe d'hommes, la bourgeoisie manufacturière, dont les assises géographiques se trouvent dans le sud de l'Ontario et dans la région de Montréal ${ }^{7}$. On peut observer cette évolution du capitalisme com-

6 Sur l'ensemble de cette période, voir notamment les ouvrages déjà cités de Hamelin et Roby, de Skelton, de McDiarmid, de Easterbrook et Aitken. Aussi: R.E. Caves et H.R. Holton, The Canadian Economy, Prospect and Retrospect (Cambridge, Mass., Harvard University Press), "Harvard Economic Studies" vol. CXII, 1961 (c, 1959), xxii-676p.; O.J. Firestone, Canada's Economic Development, 1867-1953 (Londres, Bowes and Bowes, [1958]), 384p.; André Gosselin, "L’évolution économique du Québec: 1867-1896", dans Economie québécoise (Les Presses de l'Université du Québec, 1969), 105-141; André Raynauld, Croissance et Structure économiques de la Province de Québec (Québec, Ministère de l'Industrie et du Commerce du Québec, 1961), 657p.; Tom Naylor, The History of Canadian Business 1867-1914, 2 volumes (Toronto, James Lorimer \& Co., 1975).

7 Alfred Dubuc, Les classes sociales au Canada de 1760 à 1840 (Montréal, Librairie de l'Université de Montréal, 1967) a déjà souligné, dans sa discussion sur la bourgeoisie canadienne, la distinction à faire entre la bourgeoisie commerciale et la bourgeoisie industrielle; il notait que la bourgeoisie dont D. Creighton, The Commercial Empire of the St. Lawrence, 1760-1850 (Toronto, Ryerson Press, 1937) et Fernand Ouellet, Histoire économique et sociale du Québec 1760-1850. Structure et conjoncture (Montréal, Fides, [c. 1966], xxii-639p.) disent qu'elle était progressiste, dynamique, innovatrice, était en fait attardée au capitalisme commercial. L'avènement d'une véritable bourgeoisie industrielle ne semble se produire qu'à la fin du XIXe siècle. De son côté, Tom Naylor, ("The rise and fall of the third commercial empire of the St. Lawrence", dans Gary Teeple, Capitalism and the national question in Canada (Toronto, University of Toronto Press, [c. 1972]), 1-41, parle plutôt de la formation, sous le régime confédératif, d'un troisième empire commercial du Saint-Laurent, et il conclut au caractère marchand de la classe capitaliste canadienne qui s'enrichit grâce à la 
mercial au capitalisme industriel en suivant l'orientation des Chambres de commerce. D'abord fortement hostiles à tout protectionnisme, elles défendent les intérêts d'une bourgeoisie qui vit encore principalement du transport et de l'importation-exportation des marchandises. Grâce à l'arrivée de nouveaux éléments tournés, eux, vers la production manufacturière, les Chambres adoptent peu à peu des résolutions réclamant du gouvernement fédéral des mesures protectionnistes. Ainsi, la crise de 1873 mine les positions de la bourgeoisie commerciale et favorise l'ascendance du capitalisme manufacturier. Dans l'histoire économique du Canada, c'est un tournant important.

Le petit commerce canadien bénéficie jusqu'en 1873 d'une conjoncture très favorable, caractérisée par la surproduction qui pousse les producteurs et les détaillants à prendre des engagements financiers excessifs. Cette surproduction fait naître de grandes pressions incitant les producteurs et les grossistes à vendre davantage, et les marchands généraux à acheter plus qu'ils ne devraient. Ces abus aboutissent à l'accumulation de stocks énormes de marchandises dans les entrepôts et sur les étagères des détaillants. Des facteurs extérieurs expliquent cette grande activité commerciale: le Canada participe à la croissance économique de sa mère patrie politique et économique, la Grande-Bretagne, qui est au XIXe siècle la première puissance industrielle du monde. Les facteurs qui favorisent la domination de l'Europe sur le monde servent aussi à intégrer l'économie coloniale du Canada au grand marché du capitalisme européen et nord-américain. Grâce surtout aux progrès technologiques, les marchands profitent de l'expansion de l'économie européenne ${ }^{8}$.

Mais le facteur qui favorise le plus le Canada, la condition en quelque sorte de tout le reste, c'est le développement des transports: leur amélioration entraîne dans sa suite des transformations dans le système de crédit, dans la spécialisation régionale et dans l'inté-

circulation des marchandises plutôt que par la production de biens. Le caractère marchand de la bourgeoisie canadienne, selon Naylor, explique l'aspect colonial et sous-développé de l'économie canadienne dominée de l'extérieur. Dans les secteurs de la banque, des transports, de la spéculation, le passage se fait d'un capitalisme marchand à un capitalisme financier, mais non à un capitalisme industriel. De son côté, Wallace Clement, The Canadian Corporate Elite. An analysis of economic power (Toronto, McClelland and Stewart, Carleton Library, 89), xxvii-479p., distingue entre deux bourgeoisies, la canadienne et la bourgeoisie des compradors, qui ne sont que des agents de capitalistes étrangers.

8 Il existe de nombreuses synthèses sur l'évolution économique générale. Voir par exemple Frédéric Mauro, Histoire de l'économie mondiale 1790-1970 (Paris, Editions Sirey, c. 1971), 425p., et Gilbert Garrier, dir., La domination du capitalisme 1840-1914 (Paris, Armand Colin, "Histoire économique et sociale du monde" vol. 4, [c. 1978]), 624p. 
gration et l'articulation de l'économie canadienne à l'économie capitaliste occidentale. Les transactions monétaires occupent progressivement tout l'espace économique canadien. Au niveau de la vente au détail, la révolution des transports signifie un approvisionnement plus régulier, plus facile et plus fréquent auprès des fournisseurs ${ }^{9}$. C'est donc au niveau des circuits de distribution, les canaux sanguins du système économique, que la révolution des transports fait sentir ses plus grands effets dans le pays. La circulation des marchandises et des populations, au plan international ou interne, peut donc se faire avec de plus en plus de facilité.

La navigation interocéanique connaît de grands progrès au cours du XIXe siècle. Élément fondamental de l'unité de l'Empire britannique, un réseau mondial de transport maritime s'établit dans les deux premiers tiers de ce siècle, liant Londres et les grands ports anglais à l'Inde, à l'Australie et à l'Amérique du Nord ${ }^{10}$. Longtemps resté la principale force motrice, le vent est progressivement remplacé par la vapeur, surtout après le milieu du siècle. Cette nouvelle énergie motrice contribue à la baisse à long terme des coûts de fret, ce qui favorise l'expansion durant la phase $\mathrm{A}$ du kondratieff, soit de 1847 à $1873^{11}$. La navigation profite aussi des autres améliorations: des coques en fer, l'accroissement des tonnages, une plus grande rapidité et régularité des services. Ces progrès permettent aux importateurs de réaliser d'importantes économies externes. La construction de navires en fer, suite aux découvertes métallurgiques, notamment l'avènement des fours Bessemer et Gilchrist pour fabriquer l'acier, donne à la navigation à vapeur une avance définitive.

À l'intérieur du Canada, les voies de communication s'améliorent considérablement. Durant les années quarante, le réseau des canaux est complété, un peu tard il est vrai. Durant la décennie cinquante, le gouvernement canadien, encouragé par les ambitions des financiers et constructeurs anglais, aide à la construction du Grand-Tronc, chemin de fer dont la voie principale s'étend de

9 Sur l'histoire des transports au Canada, voir principalement A.W. Currie, The Grand Trunk Railway of Canada (Toronto, University of Toronto Press, 1957), viii-556r.; A. Faucher, Québec en Amérique au XIXe siècle. Essai sur les caractères économiques de la Laurentie (Montréal, Fides, [c. 1973]), xviii-247p.; G.P. de T. Glazebrook, A History of Transportation in Canada (Toronto, McClelland and Stewart, Carleton Library 11 et 12, 1962), 2 volumes; H.A. Innis, A History of the Canadian Pacific Railway (Toronto, University of Toronto Press, [c. 1971]), édition originale de 1923, xxii-365p.; G.R. Stevens, Canadian National Railways (Toronto, Clarke Irwine \& Co, 1960 et 1962), 2 volumes.

10 Par exemple, la ligne Cunard. Voir Thomas E. Appleton, Ravenscrag. The Allan Royal Mail Line (Toronto, McClelland and Stewart, [c. 1974]), xiii-222p.

11 Mauro, Histoire de l'économie..., passim. 
Sarnia, en Ontario, à Portland, dans le Maine; un embranchement relie Richmond à Rivière-du-Loup. Plusieurs lignes secondaires sont absorbées par le Grand-Tronc dont le seul rival de taille est le Great-Western, un chemin de fer qui relie Hamilton à Windsor et à Sarnia. Ce dernier menace toujours de court-circuiter le transport des marchandises entre l'Ouest américain et la côte atlantique. Vers 1870 , il est déjà beaucoup question du grand rival futur du GrandTronc, le Pacifique-Canadien, qui sera construit durant les années quatre-vingt. Le Canada jouit donc, en 1870, d'un réseau de canaux permettant de naviguer sur tous les Grands Lacs et le St-Laurent, ouvert une partie de l'année seulement, et un réseau ferroviaire de $4210 \mathrm{~km}$ (2617 milles) traversant les principales régions habitées du pays. Il manque encore l'Intercolonial pour relier le Canada central aux Maritimes, ligne qui ouvrira en 1876. Enfin, il existe un certain nombre de routes de terre ou de gravier unissant tant bien que mal, et selon les saisons, les nombreuses petites communautés rurales à leurs centres régionaux de distribution ${ }^{12}$.

Une autre découverte technologique transforme grandement l'économie: la télégraphie et le code Morse. Mise au point durant les années trente et quarante, la télégraphie, après l'installation d'un câble transatlantique à la fin des années cinquante, met les acheteurs (ou les vendeurs) canadiens en communication directe avec les fournisseurs (ou agents) britanniques. Le marchand général n'a pas l'habitude de faire ses commandes directement en Grande-Bretagne, mais il bénéficie indirectement des avantages d'un système plus efficace de commande. En 1858, le câble transatlantique reliant la Grande-Bretagne à Terre-Neuve est posé, et dans les années suivantes, le réseau télégraphique s'étend le long des voies ferrées pour recouvrir tout le Canada central.

En résumé, les transports sont le facteur critique, l'élément fondamental qui explique l'expansion commerciale canadienne vers 1870. Les voies de communication reliant le Canada à l'intérieur et à l'extérieur favorisent, plus que tout autre agent, les échanges.

\section{II- LA PRATIQUE DU PETIT COMMERCE}

Le commerce de détail, vers 1870 , est passablement différent de ce qu'il deviendra durant le siècle suivant: ni boîtes de conserve, ni emballage spécial, ni vitrines décorées, ni sacs de papier. Au XIXe

12 J.C. Lessard, Les transports au Canada (Montréal, Commission royale sur les perspectives économiques du Canada, 1956). 
siècle, les familles canadiennes produisent encore elles-mêmes une grande partie de ce qu'elles consomment. Mais à mesure que progresse l'industrialisation et que la population rurale s'intègre à l'économie de marché, il devient de plus en plus nécessaire de satisfaire les besoins de la population par un réseau étendu de distribution des marchandises. L'expansion de ce réseau répond donc à une transformation de l'économie et de la société canadiennes.

\section{A- Une typologie imprécise}

Au départ, il n'est pas facile de cerner les dimensions et les caractéristiques de la classe que nous avons appelée "les petits commerçants" ou "les détaillants". Le recensement canadien de 1871 divise la main-d'oeuvre active du pays en différentes "classes professionnelles". La population active du pays s'élève alors à 1009848 personnes. Environ $14 \%$ de celle-ci entre dans la catégorie des "non classifiés". Pour le reste, la population active se répartit dans cinq grandes classes: la classe agricole $(55,3 \%)$, la classe industrielle $(24,6 \%)$, la classe commerciale $(8,7 \%)$, la classe domestique $(6,9 \%)$ et finalement les professions libérales $(4,5 \%)$. Le tableau I montre aussi la répartition de cette population active par classes et selon les provinces, ce qui permet de constater certains écarts provinciaux: en Ontario, la classe commerciale ne constitue que $6,3 \%$ de la population active, alors qu'en Nouvellc-Écosse elle représente $11,4 \%$.

Mais que faut-il entendre par "classe commerciale"? Elle ne correspond pas très bien aux petits commerçants et détaillants qui nous occupent car elle comprend tout, depuis les comptables et les banquiers jusqu'aux cochers, encanteurs et gardiens d'estacades. Le recensement de 1871 permet cependant d'isoler de cette classe certaines catégories d'occupations. Le tableau II apporte des données sur le nombre de personnes qui se retrouvent dans diverses occupations relatives au commerce de détail. On constate qu'ensemble, ces occupations ne représentent qu'environ le tiers de la "classe commerciale". L'imprécision même des catégories crée des divergences importantes entre les provinces. Cependant, on peut penser que les totaux provinciaux fournissent une idée du nombre approximatif de marchands.

Quelques années plus tard, cependant, une liste donnée par Dun, Wiman \& Co semble contenir beaucoup plus de noms pour la 
TABLEAU

LES CLASSES PROFESSIONNELLES AU CANADA (1871)

\begin{tabular}{l|r|r|r|r|r|r|r|r|} 
& $\begin{array}{c}\text { Population } \\
\text { totale }\end{array}$ & $\begin{array}{c}\text { Population } \\
\text { active }\end{array}$ & $\begin{array}{c}\text { Classe } \\
\text { agricole }\end{array}$ & $\begin{array}{c}\text { Classe } \\
\text { domestique }\end{array}$ & $\begin{array}{c}\text { Classe } \\
\text { commerciale }\end{array}$ & $\begin{array}{c}\text { Classe } \\
\text { industrielle }\end{array}$ & $\begin{array}{c}\text { Professions } \\
\text { libérales }\end{array}$ & $\begin{array}{c}\text { Non } \\
\text { classifiés }\end{array}$ \\
\cline { 2 - 9 } Ontario & 1620851 & 463424 & 228708 & 26805 & 29082 & 93871 & 16759 & 68199 \\
Québec & 191516 & 341291 & 160641 & 21186 & 25507 & 65707 & 15376 & 52874 \\
Nouveau-Brunswick & 285594 & 86488 & 40394 & 5358 & 7081 & 18683 & 2858 & 12114 \\
Nouvelle-Ecosse & 387800 & 118645 & 49769 & 6755 & 13531 & 34547 & 4151 & 9892 \\
Total (Canada) & 3485761 & 1009848 & 479512 & 60104 & 75201 & 212808 & 39144 & 143079
\end{tabular}

Source: Recensement du Canada 1871, vol. V. tableau L. 
TABLEAU II

QUELQUES GROUPES FAISANT PARTIE DE LA “CLASSE COMMERCIALE” EN 1871

\begin{tabular}{|c|c|c|c|c|c|c|c|}
\hline & $\begin{array}{l}\text { Commis- } \\
\text { voyageurs }\end{array}$ & $\begin{array}{c}\text { Petits } \\
\text { négociants }\end{array}$ & Epiciers & $\begin{array}{l}\text { Marchands } \\
\text { forains }\end{array}$ & Marchands & Boutiquiers & Total \\
\hline & 344 & 655 & 1384 & 507 & 5839 & 1699 & 10528 \\
\hline $\begin{array}{l}\text { Ontario } \\
\text { Ouéhec }\end{array}$ & 159 & 2704 & 877 & 106 & 4451 & 458 & 8755 \\
\hline $\begin{array}{l}\text { Québec } \\
\text { Nouyeau-Brunswick }\end{array}$ & 6 & 385 & 266 & 19 & 1228 & 339 & 2243 \\
\hline Nouvelle-Écosse & 8 & 329 & 280 & 34 & 1828 & 587 & 3066 \\
\hline TOTAL & 517 & 4073 & 2807 & 666 & 13446 & $3 \overline{083}$ & 24592 \\
\hline
\end{tabular}

Source: Recensement du Canada 1871. extraits du vol. II, tableau XIV. 
TABLEAU III

LE NOMBRE DE MARCHANDS, 1877 et 1880

$\begin{array}{lrr} & 1877 & 1880 \\ & & \\ \text { Toronto (tout l'Ontario sauf dix comtés de l'est) } & 29366 & 32745 \\ \text { Montréal (tout le Québec et dix comtés de l'est de l'Ontario) } & 15559 & 14817 \\ \text { Halifax (toute la Nouvelle-Ecosse) } & 5960 & 4617 \\ \text { Saint-Jean (tout le Nouveau-Brunswick) } & 3368 & 3353 \\ \text { TOTAL } & 54244 & 55562\end{array}$

Source: Monetary Times, 30 janvier 1880, p. 89.

période 1877-188013. Ces données figurent dans le tableau III. Les chiffres représentent vraisemblablement le nombre de personnes pratiquant une activité commerciale et comprennent sans doute beaucoup de petits entrepreneurs et "contracteurs", en plus des petits commerçants proprement dits. Les données du recensement de 1871 semblent donc projeter une meilleure image de ce qu'a pu représenter la "classe commerciale" véritable en 1871 et durant la décennie suivante. Il ne nous a cependant pas été possible de découvrir quelle proportion de cette classe peuvent représenter les marchands-détaillants ou les petits commerçants. Car il faut sans doute ajouter aux "petits commerçants" proprement dits, les épiciers et un bon nombre de "marchands" et de "boutiquiers".

C'est pourtant à cette vague classe de petits détaillants et marchands que s'adresse le Monetary Times. Le journal veut parler à toutes les régions du Canada, et les analyses qu'il publie s'appliquent en principe au pays entier, mais il semble bien que le Canada central soit plus particulièrement visé dans les commentaires du journal. 


\section{B- La vie du petit commerce}

Pour réussir, le petit commerçant doit établir des relations à deux niveaux: en amont, il doit s'approvisionner chez les grossistes, les fournisseurs, les producteurs; en aval, il doit écouler sa marchandise. Le petit commerce est donc l'intermédiaire entre ces deux niveaux.

Dans un système économique, les circuits de distribution peuvent être soit directs (producteur-consommateur), soit courts (producteur-détaillant-consommateur), soit longs (producteurgrossiste-détaillant-consommateur) ${ }^{14}$. Au XIXe siècle, le réseau canadien de distribution est surtout de type long, et à l'occasion de type direct. Les grandes distances entre les producteurs et les consommateurs commandent un tel système d'au moins deux intermédiaires.

Dans l'économie canadienne d'alors, et surtout à la campagne, où vit la majorité des habitants, l'intégration de la population à l'économie de marché n'est que partielle; les Canadiens produisent une grande partie de ce qu'ils consomment. Leurs achats, faits quelquefois durant l'année, se limitent aux seuls produits qu'ils ne peuvent fabriquer, ou qui doivent être importés (thé, sucre, etc.). Avant de parvenir aux consommateurs et aux détaillants, ces marchandises ont passé entre les mains des importateurs et des grossistes. D'où viennent ces produits vendus aux consommateurs canadiens? Pendant longtemps, la Grande-Bretagne est le principal pays d'origine des importations mais elle est plus tard progressivement remplacée par les États-Unis, surtout au XXe siècle. Ce qui intéresse particulièrement les marchands, ce sont les articles divers: il semble que les articles de mercerie constituent le principal groupe des importations ${ }^{15}$.

Examinons d'abord les relations du marchand avec son fournisseur ou son grossiste. Vers 1870 , les marchands de campagne disposent essentiellement de trois moyens pour commander leurs marchandises. Ils peuvent se rendre personnellement chez le producteur ou le grossiste; ils peuvent en deuxième lieu, faire une commande par écrit ${ }^{16}$; ils peuvent enfin, en troisième lieu, passer des

\footnotetext{
14 Armand Dayan, Manuel de la distribution. Fonction, structures, évolution (Paris, Editions d'organisation, 1973), 17.

$15 M T, 11$ août $1876,158-159$.

16 Dans ses commandes aux grossistes, le détaillant ne fait pas toujours preuve de clarté, ce qui peut causer des malentendus entre les fournisseurs et les détaillants. Certains marchands commandent chez le grossiste en envoyant des billets tels que "some of the same
} 
commandes aux commis voyageurs qui circulent en nombre croissant à travers le pays. Jusqu'au milieu du XIXe siècle, la pratique courante veut que le marchand ou le détaillant de campagne se rende deux fois l'an, au printemps et à l'automne, visiter les grossistes dans les villes de Québec, Montréal, Toronto ou Hamilton. Il arrive même que le marchand ne s'approvisionne qu'une fois l'an. Les marchandises achetées sont expédiées par eau ou par terre, ou parfois même en traîneau durant l'hiver, si l'état des routes devient impraticable ${ }^{17}$. Le marchand, avec le temps, s'approvisionnera plus régulièrement, selon la qualité des transports disponibles ou selon son éloignement des centres de distribution. L'arrivée du chemin de fer, qui a sur la navigation l'avantage de fonctionner à longueur d'année, modifie en profondeur les habitudes du petit commerce en fournissant un accès relativement facile et des moyens de transport relativement peu coûteux pour les marchandises, et ce durant toute l'année.

Les commandes par écrit ont donné lieu à des abus: les grossistes, manquant parfois de scrupules, pratiquent le "stuffing", c'est-à-dire l'envoi aux marchands éloignés de plus de marchandises qu'ils n'en avaient demandé afin de bien remplir toutes les boîtes. On en profite aussi, parfois, pour envoyer des produits nouveaux ou des marchandises nouvelles. De son côté, le marchand n'est pas toujours très précis dans ses commandes. Beaucoup de ces abus cessent avec l'amélioration des moyens de transport.

Une autre pratique qui se répand chez les détaillants et les petits commerçants, vers 1870 , c'est de commander directement chez les producteurs anglais, sans passer par les importateurs ou les grossistes. L'envers de cette pratique est que les producteurs anglais prennent même l'habitude d'envoyer leurs propres commis voyageurs recueillir les commandes. Pour accommoder les détaillants, les producteurs se montrent même disposés à vendre leurs marchandises en petites quantités. Toutes ces pratiques soulèvent l'opposition du Monetary Times qui les juge néfastes et conseille de les éviter. Le journal prétend que les marchandises envoyées ne correspondent pas toujours aux échantillons montrés par le commis voyageur, et qu'il est difficile, à une si grande distance, de faire remplacer les marchandises non satisfaisantes: "Then it is not

stuff you sent me before", ou encore un cordonnier demande "a box of hardash, some shoe thread, a little good straping". Ces commandes sont difficiles à remplir, font perdre beaucoup de temps et d'énergie aux grossistes. Le Monetary Times exhorte donc les détaillants à expédier des commandes précises et explicites.

$17 M T, 2$ décembre $1870,305$. 
possible to get as good assortment for a given sum of money as can be obtained from large and regular Canadian importers." 18 Il arrive aussi que le détaillant doive acheter des quantités plus grandes que celles qu'il achèterait chez un grossiste ${ }^{19}$.

Au début des années soixante-dix, une période de production intensive modifie aussi les caractéristiques du commerce de gros. Les grossistes ont généralement en Angleterre des "resident buyers", des agents chargés de faire régulièrement les achats de leurs clients tout au cours de l'année. Les marchandises ainsi commandées sont ensuite expédiées au Canada sur plusieurs bateaux différents. $\mathrm{Ce}$ système nouveau, rendu possible par l'amélioration des moyens de transport et du télégraphe, permet au grossiste de renouveler continuellement son stock, car les commandes peuvent parvenir en Angleterre le jour même où le grossiste ou l'importateur les expédie. Les stocks étant ainsi reconstitués plus régulièrement, ils ne doivent pas être aussi considérables qu'auparavant. De plus, le grossiste peut mieux profiter des baisses de prix quand elles se produisent.

La prospérité générale, durant la période 1870-1873, aboutit à une surproduction qui amène les producteurs et les grossistes à faire de grands efforts pour trouver des débouchés à cette production. Il s'ensuit une grande rivalité qui entraîne tout le monde, depuis le producteur et le grossiste jusqu'au détaillant, à accorder trop de crédits à ses clients.

Pour les petits commerçants, qui nous intéressent plus particulièrement et qui exploitent des magasins généraux, les produits qu'ils achètent sont surtout des articles d'épicerie (épices, sel, sucre, thé, etc.), de quincaillerie, de mercerie et de pharmacie ${ }^{20}$. Par la force des choses, les marchands font donc un commerce général: dans un pays grand comme le Canada, où la population est clairsemée, il n'est pas rentable ni praticable d'ouvrir des boutiques spécialisées, sauf parfois dans les villes.

Ainsi, en amont, le marchand entretient des relations avec ses fournisseurs; mais pour réaliser des profits, le marchand doit écouler ses marchandises. Comment s'établissent ces relations entre les petits commerçants et leurs clients?

$M T, 17$ janvier $1873,598$.

$M T, 2$ décembre $1870,305$.

20 Hamelin et Roby, Histoire économique..., 345, où se trouve une liste du genre d'articles qu'un détaillant pouvait commander pour son commerce. 
Il semble que le marchandage soit assez communément pratiqué: l'idée d'un prix unique ne s'est véritablement implantée dans les moeurs commerciales qu'avec la venue des magasins à succursales. Selon Jean Hamelin et Yves Roby, c'est T. Eaton qui introduit le premier cette "révolution" au Canada: "il marque le prix sur chaque article, bannit tout marchandage, impose la vente au comptant, introduit l'échange ou remboursement des marchandises défectueuses."21 Timothy Eaton ouvre son magasin au 178 de la rue Yonge en 1869. Sa première annonce parue dans les journaux, en petits caractères, énonce sa politique: "We propose to sell our goods for CASH only - In selling goods, to have only one price". Eaton, lui, achète à crédit chez le grossiste John Macdonald ${ }^{22}$.

Pour conserver, en établissant leur prix, une idée de leur marge de profit, les marchands utilisent fréquemment un code: ils prennent par exemple une expression ou un mot de dix lettres différentes et chaque lettre représente un chiffre de 0 à 9 . Si, par exemple, le marchand utilise le mot "bastringue", la mention tti/ rig indique que le prix de revient est $\$ 3,35$ et le prix de vente $\$ 4,57$. Parfois, on utilise une onzième lettre pour signifier la répétition d'un chiffre ${ }^{23}$.

Nous connaissons peu de choses sur la comptabilité que tiennent les marchands généraux. Selon l'idée qu'en laisse le Monetary Times, elle doit être très mauvaise ou inexistante.

Le marchand peut recevoir le prix des marchandises vendues sous trois formes: en produits (le troc), en argent comptant, ou sous forme d'une créance qu'il accorde à ses clients (achat à crédit). Le troc, vers 1870, est en voie de disparition. Antérieurement, dans les colonies, le manque de numéraire a contraint les colons à payer leurs achats chez le marchand général en nature, c'est-à-dire en offrant divers produits en paiement (de la potasse, du beurre, éventuellement du bois ou du blé). Ce système a fleuri dans certains milieux au début du XIXe siècle, principalement à la campagne où les marchands ruraux acceptent des colons les produits qu'ils expédient ensuite vers les villes. Si le mauvais état des communications a rendu le troc nécessaire ou inévitable, si le manque de numéraire l'a rendu souhaitable, il n'a plus sa raison d'être en 1870, et le Monetary Times qualifie sa continuation de "ridiculous folly". On le pratique encore, apparemment, surtout pour le beurre et la laine. Le journal prétend que le marchand et le fermier peuvent

21 Hamelin et Roby, Histoire économique..., 348.

22 Stephenson, The Store that Timothy Built, 22-23.

23 Pour une description de ce système, voir Johnson, Over the Counter..., 15. 
facilement écouler leurs produits en les vendant à des acheteurs spécialisés, sans encombrer les marchands généraux qui, pourtant, se laissent souvent tenter par des occasions de spéculer sur ces produits:

But those who set out as strorekeepers merely, often become speculators instead. From simply exchanging goods at a profit for these products, they come to look for profit to speculative ventures in what they buy from farmers rather than in their legitimate business. ${ }^{24}$

En arrêtant la pratique du troc, le marchand ne serait donc plus soumis à la tentation de spéculer.

L'achat en argent comptant semble être plus l'exception que la règle ${ }^{25}$. Il faudrait sans doute retrouver des comptabilités de marchands pour en avoir une idée plus précise et pour connaître quel pourcentage de leurs ventes est payé comptant. À lire le Monetary Times, il semble bien que l'achat à crédit soit la forme habituelle de paiement.

Quels peuvent être les profits d'un marchand général? Nous n'avons pas trouvé de renseignements précis sur cette question capitale: des monographies seraient indispensables pour éclairer cette dimension du problème. Le Monetary Times indique que les profits sont élevés sur les objets de luxe, mais faibles sur les articles ayant un usage plus ordinaire ${ }^{26}$. Cependant, ces renseignements ne disent rien sur les taux de profits que peut espérer un marchand moyen, bien qu'il semble que la situation des détaillants se détériore au cours de la décennie. Selon un lecteur du journal qui signe "Plain Dealer", il est "next to impossible for a retailer in Canada to make a decent living in these days, profits have gone down so low, and expenses are so out of proportion to them". À cette plainte, le journal répond que les "shop-keepers, as well as other people, have become wasteful and imprudent where their fathers were cautious and saving" 27 , reprenant un thème fréquent de ses colonnes.

La grande solution que le journal ne manque jamais une occasion de préconiser, c'est de devenir "systématique": les marchands doivent le devenir au niveau des achats (et n'acheter que ce

$24 \quad M T, 27$ juin 1873, 1155-1156.

25 Louise Dechêne a noté le même phénomène dès l'époque du régime français au Canada. Voir Louise Dechêne, Habitants et marchands de Montréal du XVIIe siècle (Montréal, Plon, "Civilisation et mentalités", [c. 1974]), 183-203.

26 MT, 15 octobre $1874,430$.

27 $M T, 15$ août $1879,209$. 
qu'il faut et que ce qu'ils peuvent payer), au niveau des ventes (organiser une tenue de livres, ne pas faire trop de crédits, bien gérer les stocks), au niveau du crédit (ni trop emprunter, ni trop prêter). Le marchand ne doit vendre que de la marchandise appropriée, de bonne qualité, et qu'aux bons clients. Et en tout, la sobriété et l'économie. Le journal répète très souvent ces conseils à ses lecteurs.

Les achats à crédit semblent représenter un fléau particulièrement coûteux pour les petits commerces, et le Monetary Times ne cesse d'en dénoncer les abus.

\section{C- Le système de crédit}

Il est aujourd'hui courant de décrier les vices de la consommation et d'en voir la cause dans les abus et les excès d'un système trop abondant de crédit; on dénonce les cartes de crédit, les sociétés de prêt, etc. Un peu de recul historique fait cependant voir que la situation n'est pas tellement différente vers 1870 . À un siècle d'intervalle, les deux situations présentent de troublantes ressemblances; les deux situations ne se distinguent que par une différence de degré, non de nature. Les acheteurs du XIXe siècle semblent s'être laissé aussi facilement séduire que nos contemporains par l'appât du crédit facile. On semble en avoir fait un usage très répandu, tant chez les consommateurs que chez les marchands ou même chez les grossistes.

Dans le monde des affaires, le crédit est un instrument dont on ne saurait se passer; une colonie comme le Canada possède une longue histoire pour le lui rappeler. Sans les crédits, souvent très longs, que lui accordent les fournisseurs ou des agents dans la métropole, l'activité commerciale de la colonie serait réduite à néant: “... it comes up at every turn in the wheel of business, its ramifications are so nearly endless, and, moreover, we are all so accustomed to it..."28 Autrefois, les marchands ont été amenés à faire crédit aux fermiers parce que la lenteur des moyens de communication pouvait exiger des années avant que la production d'une ferme ne rapporte de l'argent comptant, entre le moment de la récolte et sa vente en Europe ou ailleurs.

De longs crédits ont ainsi permis aux marchands, au début du XIXe siècle, d'acheter des marchandises qu'ils ne repayent que douze ou dix-huit mois plus tard. L'hiver rendant l'intérieur du pays inaccessible, le marchand général s'approvisionne durant l'été ou 
l'automne en préparation du printemps suivant. Pour répondre à ces besoins, les maisons canadiennes de gros doivent donc, elles, faire leurs achats l'année précédente. Généralement, un acheteur représentant le grossiste fait durant l'été la visite des producteurs anglais et achète d'un seul coup les stocks nécessaires pour une année; le grossiste les revend à l'automne ou au printemps suivant ${ }^{29}$.

Si on en croit le Monetary Times, les coutumes ont la vie dure et ne changent que difficilement, et même l'amélioration des moyens de transport ne transforme pas les vieilles habitudes. Selon le journal, le système de crédit érigé autrefois n'a plus sa raison d'être. À l'époque de la Nouvelle-France, le manque de numéraire avait amené les intendants, à quelques reprises, à fabriquer leur fameuse monnaie de carte. Dans le siècle qui suit la Conquête, les billets des gros marchands et les billets de l'armée (les "army bills") circulent comme monnaie. La création de banques, dans la deuxième décennie du XIXe siècle, a cependant mis en circulation une certaine quantité de billets de banque ${ }^{30}$. Pourtant ces deux facteurs, l'amélioration des moyens de transport et la circulation de la monnaie, n'éliminent pas le recours aux longs crédits, devenus moins nécessaires. À mesure que l'économie canadienne se modernise et s'intègre au capitalisme occidental, la monnaie circule davantage. Pourtant, les marchands continuent d'acheter à crédit.

Le Monetary Times nous renseigne aussi sur la manière dont fonctionne ce système de crédit. Par exemple, quand un marchand importateur achète à Liverpool des marchandises d'une valeur de $£ 1000 \mathrm{stg}$, il achète le tout à crédit ("since he will sell on credit, he buys on credit"). La marchandise sera éventuellement vendue aux marchands généraux pour $£ 1000$ stg plus les coûts de transport, d'assurance, de douane, etc., plus une augmentation suffisante pour couvrir les mauvaises créances ainsi que les profits anticipés par l'importateur. Pour payer ses achats auprès des fournisseurs, le petit commerçant ou marchand général leur donne des billets à ordre ("promissory notes") escomptés par les banques après l'endossement de l'importateur. La banque escompte ces billets de manière à obtenir un taux d'intérêt de $7 \%$ par année sur ce billet, plus une autre somme parce qu'il s'agit d'un effet à long terme. L'argent ainsi obtenu est versé à l'importateur sous forme d'un billet à terme en

$29 \quad M T, 2$ décembre $1870,305$.

30 Sur l'histoire des banques canadiennes, voir E.P. Neufeld, The Financial System of Canada. Its growth and development (Toronto, Macmillan, [c. 1972]), 645p.; R. Craig McIvor, Canadian monetary, banking and fiscal development (Toronto, Macmillan, 1958), xix-263p. 
livres sterling ("sterling bill drawn on time"), sur lequel la banque réalise un nouveau profit. Tous ces frais, éventuellement, retombent sur le prix des marchandises et on a bien tort de croire, ajoute le journal, que les importateurs défraient ces coûts car, ultimement, ce sont les clients qui couvrent tous les coûts ${ }^{31}$.

Le système de crédit affecte tous les secteurs, mais il cause de pires dégâts dans certains secteurs:

The system is very much more pernicious in the grocery than in the dry goods trade, for the profits on groceries are so much smaller that it is frequently a source of loss to the merchant to allow his customers to run an account for three, six, or nine months, unless he judiciously charges them a little more for every article they buy, in order to make up for the time he has to remain out of his money. ${ }^{32}$

Les abus semblent tout aussi répandus chez les petits boutiquiers tels que les tailleurs, les cordonniers et les autres. Si par hasard un marchand a recours à une agence de perception, celle-ci impose de toute manière des frais de $5 \%$ et $10 \%$. Or les marges de profit sont beaucoup trop minces pour supporter de tels frais. Quant au petit commerçant dans un village, il lui est évidemment très difficile d'accorder du crédit à tel client et de le refuser à tel autre.

Durant la période de prospérité des années 1870-1873, on peut s'étonner que les fermiers aient tant recours au crédit au lieu de payer leurs achats comptant. La mauvaise habitude plutôt que la nécessité explique sans doute ce phénomène; ou peut-être les fermiers, meilleurs hommes d'affaires qu'on ne le suppose, croientils préférable de retarder aussi longtemps que possible le paiement d'une dette sur laquelle ils ne payent pas d'intérêt! Dans une lettre au rédacteur du Monetary Times, un commentateur signant "A Mercantile Reporter" affirme que la classe agricole est la plus riche. Les fermiers vendent leurs produits et obtiennent de l'argent comptant qu'ils déposent dans les banques, alors qu'ils achètent leurs vêtements, leurs aliments et autres nécessités à crédit de un, deux ou trois ans sans avoir à payer d'intérêts:

Under these circumstances is it to be wondered at that the retailer is nearly all the time hard up, and continually asking renewals or a compromise. I can point to dozens of cases where country store keepers claiming to be worth four or five thousand dollars have over twice that amount in outstandings. ${ }^{33}$

$31 \quad M T, 28$ avril $1871,726$.

$32 M T, 29$ août $1873,201$.

$33 M T, 11$ septembre 1874, 295. 
Les petits commerçants sont ainsi contraints d'accorder à leur tour de longs crédits parce que leurs concurrents en font autant. L'auteur de cette lettre suggère que les détaillants se concertent pour fixer en commun un délai maximum et propose même de réunir les commerçants en congrès pour lutter contre "this large and growing evil - long credit" 34 .

Le Monetary Times est tout à fait en accord avec une politique de paiement comptant:

In the early settlement of the country, long credits were unavoidable. These were the days when there was but little wealth in the country - when no railways, and but few good roads existed - when there were but few markets, and only occasionally cash was obtained for produce; during the early period the settlers saw very little money, and unless they bought on a long time, the great majority of them could not buy at all. But this condition of things has long since passed away. Wealth has become abundant, railways have brought markets within a few miles of every farmer's door; cash can be obtained for every article produced, and no excuse exists for contuinuing the ruinous system of twelve and fifteen months' credit which still obtains in some localities. ${ }^{35}$

Le journal conseille ensuite aux provinces maritimes de s'engager le plus tôt possible dans un système de paiement au comptant. Le pays étant prospère, le moment ne peut être mieux choisi pour faire cette conversion au régime du comptant. Durant des années, le journal ne cesse de préconiser ces politiques, le crédit étant devenu à ses yeux "un mal intolérable". Et, ajoute le journal, le mal est pire en Ontario qu'ailleurs $^{36}$.

La facilité des crédits coûte donc cher à plus d'un point de vue. Par exemple, le fait d'accorder des crédits de longueur variable (un, deux, trois, six, neuf mois, et même un an ou plus) entraîne chez les grossistes beaucoup plus de travail de comptabilité. Outre les coûts inhérents à tout système de crédit, on doit ajouter les risques de pertes pour mauvaises créances ${ }^{37}$. Au niveau du consommateur, les coûts se traduisent par toutes sortes de charges additionnelles qui

34 Loc. cit.

$35 M T, 21$ octobre $1870,185$. 305.

36 Pour une discussion de tout ce problème voir notamment $M T, 2$ décembre 1870 ,

37 Vers 1892, selon J.E. Hansford, The Business Guide : or, Safe Methods of Business (Toronto, Coles Reprint, 1892), 276p., la pratique courante était d'ajouter de $10 \%$ à $15 \%$ au prix des marchandises, ce qui couvrait non seulement les intérêts, mais aussi les pertes imputables aux mauvaises créances des marchands. 
s'incorporent au prix de vente; pour le marchand, les coûts augmentent le prix des marchandises achetées. Les grossistes et les importateurs sont à leur tour obligés de s'endetter envers les producteurs qui, eux, préfèrent vendre à crédit plutôt que de ralentir ou arrêter leur production. Ainsi s'établit un long circuit allant du producteur au consommateur, un enchaînement de crédit qui lie chaque niveau de distribution à son niveau inférieur et à son niveau supérieur. Chacun a un intérêt manifeste à ce que le système soit maintenu. En fin de compte, tous finissent par payer plus cher, en vivant et en consommant au-delà de leurs moyens. Les fermiers, par exemple, ont généralement une dette équivalente à la valeur de leur prochaine récolte, qui se trouve ainsi hypothéquée en faveur du marchand local. Il suffit alors d'une mauvaise récolte, d'un accident climatique, et tout le système risque de s'effondrer. Le fermier doit alors demander des délais simplement pour assurer sa consommation courante:

When this state of things becomes wide-spread there is a general stoppage of remittances; the whole machinery of credit, running through retail and wholesale merchants, manufacturers, bankers, everybody, is put out of gear... taking the accumulations of these debts on the average, not more than 50 per cent can be realized out of them. The fact then appears that this is a form of credit which is not profitable and satisfactory to the creditor, and therefore ought not to be continued. ${ }^{38}$

La concurrence, dans ces années de prospérité, oblige cependant les marchands, les producteurs et les grossistes à défendre leurs intérêts et à limiter la conduite généreuse de leurs concurrents, en accordant autant de crédit qu'eux. Ainsi, tout conspire à engendrer des abus; la "main invisible" si chère aux économistes libéraux attend cependant l'occasion de rétablir la situation.

Ce régime de crédit conduit à un autre abus, celui des stocks excessifs de marchandises. En plus de s'endetter, le marchand général constitue pour son commerce des stocks trop grands et composés d'articles parfois difficiles à revendre, tels les produits de luxe. Le Monetary Times voit même dans ce phénomène l'explication des importations trop abondantes et, en conséquence, de l'endettement des importateurs canadiens envers les agents et les producteurs étrangers. Quand la crise économique éclate en 1873, beaucoup de marchands ne parviennent pas à survivre en affaires à cause de leurs stocks trop considérables et impossibles à vendre, et à 
cause des dettes qu'ils ne peuvent restituer à leurs fournisseurs parce que leurs propres créances ne sont pas percevables. Seule la voie de la banqueroute reste alors ouverte.

En plus du sur-stockage, un autre mal se glisse dans le régime de crédit, le chevauchement des périodes de crédit ("overlapping credit)". Par ce processus, un petit commerçant peut, par exemple, acheter au printemps des marchandises qu'il ne repaiera qu'à la fin de l'automne ou même au printemps suivant. Entre-temps, ce même marchand fera à l'automne ses achats pour la période de l'hiver, sans avoir à acquitter ses dettes du printemps. De la sorte, le marchand peut prolonger, grâce à la longueur des délais accordés, son crédit de manière à couvrir deux périodes de commerce. Il devient ainsi possible pour un marchand d'obtenir toutes les marchandises nécessaires pour une année complète sans débourser un seul sou. Plusieurs marchands, sans doute, s'acquittent bien de leurs dettes, mais d'autres, moins chanceux ou moins scrupuleux, ferment plutôt les portes de leur commerce. Le Monetary Times recommande donc fortement aux fournisseurs de n'accepter que des billets à court terme, pour éviter "this over-lapping of credit,... a fertile source of mischief" 39 . Les désavantages de ce régime sont doubles; premièrement, deux achats (au printemps et à l'automne) se succèdent sans aucun déboursé par le petit commerçant, et, deuxièmement, cette trop grande facilité de crédit conduit les petits marchands à constituer des stocks excessifs: les marchands achètent alors au-delà de leurs besoins et, surtout, au-delà de leur capacité de payer. Ainsi, le crédit conduit autant le marchand que le grossiste à la ruine ${ }^{40}$.

Résumons les abus découlant de l'abondance des crédits: inflation des prix, sur-consommation par les clients, sur-stockage par les marchands, périodes trop longues de crédit, l'endettement à tous les niveaux sans garanties suffisantes, immobilisation du capital pour de trop longues périodes, obligation pour certains marchands de devenir semi-banquiers ${ }^{41}$.

Dans un tel régime, il est inévitable que des clients se trouvent un jour incapables de rembourser leur dette; c'est pour parer à ce danger, sans doute, que naissent à l'époque les “agences de

$39 M T, 2$ décembre 1870, 305 .

40 Loc. cit.

41 Sur le rôle essentiel du marchand de campagne dans le système de crédit, Hamelin et Roby s'expriment ainsi: "Il est plus qu'un simple intermédiaire entre les grossistes des villes et les habitants des campagnes. Il est, à toute fin pratique, par le jeu des billets et des hypothèques, le financier qui assure un crédit indispensable à l'habitant durant la saison morte et les mauvaises années" (p. 337). 
collection", organisations se chargeant de recueillir les créances en souffrance. Mais les marges de profit sont tellement faibles que ces coûts supplémentaires de perception ne peuvent pas facilement être absorbés par les marchands, car il existe des frais pour devenir membre de ces agences ${ }^{42}$.

Comment réformer ces abus? Ou plutôt, pourquoi réformer des abus dont tous semblent profiter, depuis les producteurs britanniques affamés de débouchés et disposés à accorder des crédits aux importateurs et aux grossistes, depuis ces grossistes désireux de vendre aux marchands et aux détaillants, depuis ces détaillant "sur-stockés" qui donnent du crédit à leurs clients afin d'écouler leurs marchandises, jusqu'aux consommateurs qui ne demandent pas mieux que de consommer ce qu'on leur propose. Les petits commerçants craignent qu'une baisse des crédits ne réduise le volume des ventes, mais le Monetary Times prétend au contraire que la fin de tous ces abus rétablirait une plus grande prospérité et par conséquent entraînerait une plus grande capacité pour chacun de payer ses dettes. Le malaise ne durerait que le temps nécessaire pour éliminer les anciennes dettes ${ }^{43}$. Un deuxième obstacle à ce programme de réforme concerne les grossistes qui, ayant eux aussi d'énormes stocks, ne demandent pas mieux que de vendre à crédit "so long as the banks will discount the paper representing these sales"44. Le jounal reconnaît les difficultés de changer tant d'habitudes:

We know that writing against these practices is, when measured by results, very much the same as whistling against the wind. Merchants will sell and sell, trust and renew, just for the sheer force of habit, however vicious the system may be known to be. ${ }^{45}$

Les réformes précises dont le journal se fait l'avocat se résument à ceci: premièrement, dans le cas où il est indispensable de recourir au crédit, et compte tenu de l'amélioration des transports et des communications, l'échéance des billets acceptés par les grossistes doit être réduite à une durée plus raisonnable; deuxièmement, et d'une manière plus générale, partout où la chose s'avère possible, il faut revenir au système du paiement en argent comptant, le seul moyen d'éviter les abus qui accompagnent nécessairement les excès de crédit. Ces réformes, pense le journal, réduiront les prix, créeront de plus grandes liquidités, et on brisera ainsi le cercle vicieux de trop

\footnotetext{
$42 \quad M T, 30$ avril $1875,1229-1230$.

$M T, 15$ août 1873,152 .

Loc. cit.

$M T, 2$ décembre $1870,305$.
} 
acheter, trop risquer, trop s'endetter, trop stocker. Parce qu'une grande partie de leurs profits provient du commerce de détail rural, les banquiers seront les premiers à regretter ces réformes ${ }^{46}$. Mais ils ne seront pas les seuls:

The change would be most ungracious for lawyers, official assignees, bill-brokers and note-shavers; but it is useless to be unduly sympathetic towards, or shed tears over these classes; they are generally well able to take care of themselves. Neither would the banks suffer, for there are abundant opportunities for the profitable employment of their capital. The saving to the community would be immense. ${ }^{47}$

Ces lignes trahissent bien le mépris du journal pour certains groupes sociaux et sa préférence pour les petits hommes d'affaires contre les banques.

Mais d'autres facteurs que le crédit contribuent aussi à surchauffer les activités commerciales: les commis voyageurs qui deviennent à cette époque de plus en plus nombreux.

\section{D- La multiplication des commis voyageurs}

La pression, qui favorise tant l'extension d'un vaste réseau de crédit, aide aussi au développement d'un autre phénomène appelé à un grand essor dans les décennies suivantes: le commis voyageur. Dans l'imagerie populaire de l'Amérique du Nord, ce personnage occupe une très grande place. Les marchands et les boutiquiers, de tout temps, ont considéré comme des effrontés les colporteurs qui vont directement solliciter la clientèle à domicile: ce sont des rivaux traditionnels ${ }^{48}$. C'est à cette très ancienne opposition que se heurtent les commis voyageurs quand ils font leur apparition sur la scène des affaires. On avait connu dans les campagnes les "coureurs de côte", les "peddleurs", en rivalité avec les marchands. Cependant, le commis voyageur n'entre pas en concurrence avec le petit commerçant pour la vente au détail: il concurrence plutôt les grossistes. Leur importance ne déclinera qu'au XXe siècle, après l'expansion des nombreux magasins à succursales. Mais, vers 1870 , c'est un groupe nouveau en pleine croissance.

$46 \quad M T, 28$ avril $1871,726$.

47 Loc. cit.

48 Voir Abel Chatelain, "Pour une histoire du petit commerce de détail en France. Lutte entre colporteurs et boutiquiers pendant la première moitié du XIXe siècle", dans Revue d'histoire économique et sociale, XLIX, 3 (1971): 359-384. 
Le commis voyageur est un agent de commerce qui visite les détaillants pour obtenir, en faveur d'une compagnie, d'un producteur ou d'un grossiste, des commandes de marchandises. Il fonctionne habituellement à commission, d'où son intérêt évident à vendre le plus possible. En notant "the enlargement of their functions among us at the present", le Monetary Times $^{49}$ rapporte en 1871 qu'ils viennent de se regrouper dans une association canadienne des commis voyageurs. Le journal s'intéresse beaucoup à cette nouvelle profession dont il suivra d'un oeil sourcilleux l'évolution. En 1860, les États-Unis comptent 60000 commis voyageurs et ils en compteraient 200000 en 1883. Ceux-ci constituent le pilier de l'industrie hôtelière. Jusque dans les années soixante-dix, ils sont contestés aux États-Unis et l'opposition du Monetary Times à leur prolifération reflète probablement l'attitude de certains périodiques américains, comme c'est souvent le cas ${ }^{50}$.

$\mathrm{Au}$ Québec, les commis voyageurs apparaissent durant les années cinquante ${ }^{51}$. Bien que le recensement canadien de 1871 ne donne que 517 commis voyageurs dans le pays, ils auraient alors été au nombre de 1500 selon le Monetary Times. En 1890, ils seraient 2050 (dont 350 Canadiens français), ces chiffres représentant les membres de la "Dominion Commercial Travellers Association" 52.

Après la venue des commis voyageurs, le cycle saisonnier des achats subit des modifications et les marchands s'approvisionnent plus fréquemment. À la veille de 1873 , certains producteurs européens sont si pressés de vendre qu'ils envoient directement des commis voyageurs recueillir les commandes. Ces pratiques mécontentent énormément les maisons canadiennes d'importation et de gros qui se voient menacées de perdre des ventes à cause de ces intrus étrangers; elles commencent donc elles aussi à envoyer leurs agents $^{53}$. Le Monetary Times se plaint de la pratique des maisons anglaises:

A good number of retailers, many of whom are of the better class, in the country towns and villages, instead of buying from large importing houses of Montreal, Toronto, \&c., now import

$49 \quad M T, 21$ avril $1871,707$.

so Pour le point de vue américain sur ces questions, voir Johnson, Over the Counter..., 38-39.

51 Hamelin et Roby, Histoire économique..., 349.

$52 \quad M T, 24$ janvier 1890, 894.

53 Hamelin et Roby indiquent que les agents des grossistes se rendaient aussi dans les campagnes pour acheter des produits, continuant ainsi la tradition des "coureurs de costes" du régime français. Histoire économique..., 344. 
direct from Great Britain. This mode of conducting their trade has not been adopted at their instance, but is a result of that excessive competition to the evils of which the dry goods trade seems particularly subject. A certain class of English and Scotch houses, competing for the Canadian trade, send out their travellers in great numbers, and they have reduced matters to so fine a point, that a merchant here can buy from them any quantity however small. These goods are drawn against, and the effect is that the British firms engaged in that kind of business are carrying the paper of retail tirms distributed all through our towns and cities. ${ }^{54}$

Cette pratique d'envoyer des commis voyageurs montrer les nouvelles marchandises et prendre les commandes, semble se généraliser au début des années soixante-dix. Les grossistes des provinces centrales du Canada ont les premiers envoyé des commis voyageurs vendre dans les campagnes; pour ne pas laisser ceux de Montréal et de Québec s'emparer de tout le commerce, certains grossistes de Saint-Jean (NB) et de Halifax ont tôt fait de leur emboîter le pas.

Ainsi, la concurrence se pratique à deux niveaux: entre les maisons britanniques et les maisons canadiennes d'une part, et entre les maisons canadiennes des différentes villes d'autre part ${ }^{55}$. Les pauvres marchands ruraux sont parfois assaillis par les nombreux commis-voyageurs qui, pour vendre toujours plus, offrent des conditions de crédit très avantageuses. À peine leur oppose-t-on parfois une faible résistance sous forme de taxes spéciales à payer ${ }^{56}$. Le Monetary Times reproche à tous ces commis voyageurs d'exercer des pressions excessives sur les petits commerçants, et de manquer généralement de compétence et de formation pour ce travail: "Selling goods by travellers is overdone to death, from Windsor, Ont. to Sydney, C.B." 57 Le journal reproche aux commis voyageurs de n'avoir aucun intérêt dans les villages qu'ils visitent et de ne se préoccuper que des ventes et non des besoins des clients. En période de ralentissement ou de crise, les grossistes décident parfois d'envoyer un jeune commis ("junior salesman" dit le journal), une valise d'échantillons à la main. Sur la foi d'opinions aussi peu sûres, les grossistes expédient ensuite des marchandises. Selon le journal, les maisons de gros commettent une erreur et font de fausses économies en embauchant "cheap men for this important business", c'est-à-dire des jeunes commis sans expérience, ou encore des jeunes

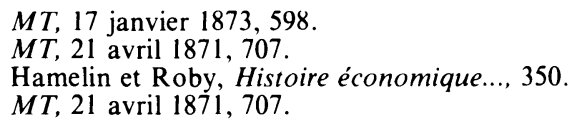


sortant à peine des collèges, quand ce n'est pas ce que le journal appelle des "broken-down country traders" qui, ayant ruiné leurs propres affaires par indolence ou ignorance, reçoivent des valises d'échantillons et deviennent par magie des dispensateurs de crédit. Exacerbé, le Monetary Times conclut en disant que les abus sont si grands que "there is a strong feeling that the abandonment of the whole system would be a wise step" 58 .

Le journal envisageait, en effet, le rôle du commis voyageur dans un tout autre sens. En plus de vendre, il ferait à l'occasion des inventaires de marchandises, analyserait les livres de comptabilité, donnerait des conseils aux marchands en difficulté financière. L'exemple vient de la Grande-Bretagne où les commis voyageurs sont

... as a rule, men of middle age, well versed in business, sound in judgment, and many of them are cultivated gentlemen besides... Nor must we look to the "drummers" of the United States for a pattern. ${ }^{59}$

Entre ces deux extrêmes, britannique et américain, il y a place pour un juste milieu canadien! La compétence doit reposer sur une connaissance des affaires et des procédés de fabrication des marchandises vendues, une connaissance technique du secteur économique où ils oeuvrent. En plus, ces commis voyageurs devraient pouvoir évaluer la fiabilité du client et posséder une expérience du pays ou au moins de la région qu'ils desservent. Enfin, ils doivent jouir d'un bon sens des affaires. C'est un programme ambitieux.

Par ailleurs, les commis voyageurs comptent aussi leurs défenseurs, comme celui qui répond en 1877 que sa profession est devenue indispensable au bon fonctionnement du commerce. Les commis voyageurs sont devenus un rouage nécessaire du commerce, au même titre que les agences de crédit, le télégraphe, les rapports commerciaux, etc. D'abord regroupés en 1871 , les voyageurs de commerce tiennent en 1873 leur première assemblée annuelle et fondent la "Commercial Travellers Association", laquelle compte alors quatre cents membres. Des exécutifs sont élus pour Hamilton, Toronto, Montréal. "It was abundantly apparent that commercial travelling has become a permanent institution" écrit le Monetary Times, ajoutant que lors de la réunion, il y avait 
trop de politique et pas assez d'affaires. Quant aux commis voyageurs, ils ne trouvent rien de mieux, pour justifier leur profession, que de citer l'Ancien testament et de se donner pour précurseur Jacob qui a envoyé ses fils en Égypte pour y acheter des céréales ${ }^{60}$ !

\section{III- LE POIDS DE LA CONJONCTURE}

Ce que la crise économique de 1873 révèle, ce sont les faiblesses fondamentales du secteur du petit commerce et la fragilité des structures. La crice devient donc l'occasion de repérer les points faibles du système général de distribution, notamment dans le petit commerce. Nous examinerons principalement les faillites. Comme le succès, en affaires, se mesure par le taux de survivance, le nombre des faillites sert d'indicateur de la santé d'un secteur de l'économie.

En septembre 1873, la panique financière éclate à New-York, puis se répercute rapidement dans toute l'économie nord-atlantique. Elle initie la phase B du kondratieff des chemins de fer, caractérisée surtout par une baisse à long terme des prix et marquée en même temps, chez les puissances européennes, par la poursuite fébrile de nouveaux marchés, ce qui les conduit à se constituer de nouveaux empires coloniaux en Afrique et en Asie et à étendre leurs zones d'influence économique sur les autres parties du monde. Au Canada, les effets de la crise ne se font sentir qu'à partir du début de 1874. Ayant prédit le malheur pendant des années, le Monetary Times a enfin raison : les faillites se multiplient, de nombreux marchands sont réduits à la ruine à cause de leurs stocks excessifs, de leurs dettes trop considérables et de mauvaises créances qu'ils ne peuvent recouvrer. La baisse des ventes, chez les détaillants, entraîne aussi une baisse des importations.

Un des signes les plus sûrs du malheur qui frappe le petit commerce à partir de 1874 , et qui s'abat sur tous les secteurs de l'économie, c'est le nombre des faillites. Parmi les domaines touchés, selon le Monetary Times, le secteur des "dry goods" est le plus atteint; le journal commente le fait avec philosophie: "the shores of the stream of trade are strewn, with the wrecks of off-hand dashing Canadian merchants of the past ten years, whose hopes were once as bright and whose hearts beat just as high as those of any enterprising dealer of to-day." 61 En 1883, devant une

$M T, 2$ janvier $1874,632$.

${ }^{61} M T, 20$ avril $1877,1204$. 
nouvelle liste de faillites, le journal en profite pour énumérer les causes des échecs commerciaux: les pertes dues à de trop longs crédits et l'ignorance de la comptabilité, l'ignorance ou le manque de compréhension des affaires, le manque de capital ou de ressources, l'encombrement de la branche commerciale, l'extravagance dans les dépenses de la famille du marchand, enfin la volonté de frauder ses créanciers. Un marchand qui veut s'assurer du succès doit connaître les affaires, disposer de capitaux, entrer dans un secteur non-encombré et fonctionner sur une base de paiements comptants ${ }^{62}$.

Au moment de la crise, vers 1874 , le journal attribue plutôt la cause des faillites à deux facteurs principaux: une mauvaise gestion des affaires (et, sous ce titre, il faut inclure les abus de crédit, la mauvaise tenue de livres, etc.); et l'encombrement dans la profession des petits commerçants.

La mauvaise gestion serait la cause de beaucoup de faillites. Le Monetary Times décrie ce qu'il qualifie de "wretched want of system in carrying business on". Il a observé ce vice autant chez les détaillants que chez les grossistes. Il blâme en particulier l'absence de comptabilité, les affaires se faisant à la bonne franquette, sans tenue régulière de livres ${ }^{63}$. Le journal canadien semble s'être souvent inspiré de publications semblables des États-Unis. Ainsi, on le voit souvent citer, par exemple, le American Grocer. C'est peut-être à ce dernier qu'il faut attribuer la préoccupation du Monetary Times pour la tenue de livres par les petits commerçants. Selon Johnson ${ }^{64}$, dès sa fondation en 1869, le American Grocer insiste sur l'importance pour les marchands de tenir des livres de comptabilité. À partir de 1874 , il recommande même de les tenir à entrée double. Cette pratique semble avoir été exceptionnelle alors, même chez les gros marchands. Chacun semble tenir ses livres comme il peut et se débrouiller tant bien que mal. Mais le Monetary Times y voit une source de bien des malheurs:

Mere jotting down of goods held on credit, is only a miserable apology for such a record of every business conducted on credit requires. ${ }^{65}$

S'il n'est pas indispensable que chacun paye un teneur de livres, il est cependant nécessaire de bien conserver des dossiers, contenant les

$M T, 5$ janvier 1883,748 .

$M T, 13$ novembre 1874,542 .

64 Johnson, Over the Counter..., 15-16.

65 $M T, 13$ novembre 1874,542 . 
renseignements précis sur tous les achats, sur toutes les ventes et sur ses droits à des remboursements. Le marchand devait ainsi noter systématiquement la date du remboursement de chaque billet, pour réduire le taux des faillites qui est alors de $20 \%$ :

For nothing can be more certain than that regular and systematic book-keeping will bring about systematic buying and selling, and systematic expenditure at home. It is folly indeed for a retailer to say he has no time to mind his books. He might as well say he has no time... to calculate at what price he should sell his goods. ${ }^{66}$

Enfin, le journal conclut que les grossistes devraient envoyer des états de compte à chaque six mois et donner de bons conseils aux détaillants.

La mauvaise tenue de compte entraine chez les petits commerçants de graves conséquences; la première est qu'on ne fait jamais de bilans financiers. En somme, on ignore toujours quels sont les profits, et on ne dispose d'aucun moyen pour gérer les dépenses. Pour connaître les profits, il faudrait faire un inventaire, ce qu'on ne fait pas non plus. Les marchands semblent faire tous leurs calculs de rentabilité à l'oeil. Les dangers d'une telle approche sont nombreux, et nota mment celui de croire à des profits souvent plus apparents que réels. Un marchand a souvent tendance à surestimer ses profits. Donc double lacune: absence de bilans et absence d'inventaires. Le résultat, c'est que les dépenses se font à partir de fausses évaluations de profits ${ }^{67}$.

Quand viennent les faillites, on découvre que la valeur de tel commerce n'est qu'une fraction de ce qu'on supposait. Sans tenue de livres, les mauvaises dettes ne sont jamais effacées (bien qu'elles soient nombreuses dans un régime où le crédit se donne avec tant de facilité), la dépréciation du capital n'est jamais calculée, enfin on se triche soi-même en gonflant la valeur des marchandises en stock: "It seems astounding how men will close their eyes to what is the actual state of things...' ${ }^{68}$ C'est ainsi que certains marchands, sans jamais tenir de livres, ont pu pendant des années faire un commerce important, achetant à crédit, donnant et recevant des billets sans les consigner dans aucun livre, sans jamais faire d'inventaires.

Pour remédier à cette situation lamentable, le Monetary Times propose la solution suivante:

66 Loc. cit.

67 Loc. cit.

$68 M T, 19$ décembre $1873,586$. 
Let our banking institution, large importing houses, etc., who have to give credit to wholesale merchants and manufacturers, make a point of requiring the production, at least once a year, of a statement of assets and liabilities. ${ }^{69}$

Le journal suggère particulièrement d'analyser, dans ces états financiers, les comptes courants exigibles (open accounts due), "for it has been proved by long experience, that most misleading errors may lurk under this simple looking item". De là, il s'ensuit que pour les détaillants, "the same suggestion is made, to be acted on even more rapidly". Si un marchand demande du crédit bien que la somme de son passif dépasse son actif et n'offre que des biens immobiliers en garantie, le grossiste devrait alors fermer le compte. En résumé: "Never economize a book-keeper."70

Une deuxième cause importante des faillites est le sempiternel problème du crédit. Le cheval de bataille que le journal enfourche à toutes les occasions réapparait ici: en 1877 , le journal écrit: "It is not too much to say that the whole of the disasters of the past two years have arisen from the abuse of credit. Credit, as everyone knows, is abused both by taking too much of it, and by making it too long." 71 Un effet secondaire bénéfique de la grande dépression de 1874-1878 est justement d'avoir en général réduit la durée des crédits accordés aux marchands ${ }^{72}$. Mais c'est une politique encore loin d'être universellement suivie, bien que les manufacturiers et les grossistes semblent s'orienter dans cette voie ${ }^{73}$.

Le nombre des faillites est une source statistique très intéressante pour l'étude de l'histoire économique du Canada. L'avènement des agences commerciales, récoltant régulièrement des informations sur la situation financière de presque tous les hommes d'affaires et commerçants du pays, nous apporte également des statistiques sur le nombre des faillites qui se produisent chaque année. Les listes de Dun, Wiman \& Co, publiées assez régulièrement dans les colonnes du Monetary Times, nous révèlent une des conséquences de la grande dépression sur les petits commerçants, c'est-à-dire sur le nombre d'entre eux que la faillite élimine chaque année.

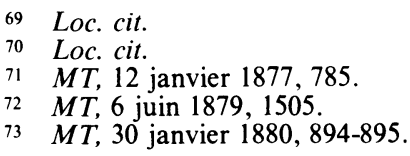


En 1882, le taux des faillites au Canada est de 1 sur 77 alors qu'il n'est que de 1 sur 122 aux États-Unis ${ }^{74}$. En 1878, le taux aurait même atteint 1 sur $33^{75}$, ce qui laisse supposer que plus de $3 \%$ des commerçants font alors faillite chaque année. Nous ignorons les taux des années précédentes.

Le nombre des faillites, d'après les chiffres de Dun, Wiman \& Co, tels que publiés dans le Monetary Times, fait voir le poids de la conjoncture et les effets de la crise de 1873

TABLEAU IV

LES FAILLITES AU CANADA, 1873-1885, SELON DUN, WIMAN \& Co. Année Nombre des faillites

Valeur des faillites (\$)

\begin{tabular}{rrr}
\hline 1873 & 994 & \multicolumn{1}{c}{12334000} \\
1874 & 966 & 7696000 \\
1875 & 1968 & 28843000 \\
1876 & 1728 & 25517000 \\
1877 & 1892 & 25523000 \\
1878 & 1697 & 23908000 \\
1879 & 1902 & 29347000 \\
1880 & 907 & 7988000 \\
1881 & 635 & 5751000 \\
1882 & 787 & 8587000 \\
1883 & 1379 & 15872000 \\
1884 & 1308 & 18939000 \\
1885 & 1247 & 8743000 \\
\hline
\end{tabular}

Source: Monetary Times, passim.

Les faillites les plus nombreuses se produisent apparemment chez les nouveaux commerçants, et non parmi les anciennes maisons, les victimes se trouvant chez ces "new houses too ambitious to become rich, or firms with inadequate capital or experience who yielded to the importunities of foreign dealers and bought too largely"76. Un résultat bénéfique de ces pertes, c'est de clairsemer les rangs trop remplis des commerçants. Ainsi, entre 1875 et 1880, les faillites rayent environ 10000 noms des listes de commerçants, aussitôt remplacés, il est vrai, par de nouveaux aspirants ${ }^{77}$.

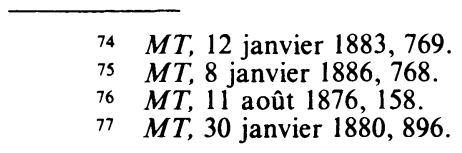


Ces faillites sont donc un phénomène important affectant le petit commerce. La conjoncture économique met en lumière les faiblesses structurales du secteur de la vente au détail.

Un deuxième aspect du petit commerce que la crise met en évidence, c'est l'encombrement de ce secteur. C'est une idée reçue que les Canadiens français encombrent les professions libérales au XIXe siècle et qu'ils hésitent à se joindre au monde économique. Ce poncif impliquerait donc que le Canada français a produit trop de professionnels et pas assez de commerçants et d'hommes d'affaires. Mais, d'après le Monetary Times, le mal de l'encombrement sévit encore plus dans le secteur du petit commerce, et la crise a au moins cet avantage d'éliminer les éléments les plus faibles de cette activité. Ce processus d'élimination a ses bons côtés, même si, selon le journal, il reste encore trop de marchands ruraux :

There are doubtless too many still in business particularly among the class called country storekeepers: but the evil has been considerably lessened... ${ }^{78}$

Dès 1874, le Monetary Times écrit qu'il existe trop de détaillants, surtout de ce type, qui n'ont pas de préparation pour le métier et qui connaissent mal le monde des affaires. D'où viennent tous ces indésirables?

We believe that if all retailers who have given up farming to go into storekeeping would retire and go back to the land, it would prove an immense service to all parties. And why not? We cannot have too many farmers. There is no keen competition in that business. Every acre cleared is so much added to our real wealth; every bushel grown is so much more actual property. ${ }^{79}$

Un journal canadien-français qui en dirait la moitié serait vite qualifié "d'agriculturiste", jugé coupable de préconiser le retour à la terre et d'avoir une mentalité conservatrice. Quant au Monetary Times, il ne manque pas de citer le cas de fermiers aisés qui, hypothéquant leurs propriétés, se lancent dans le commerce mais, dépourvus d'expérience et de savoir-faire, doivent tout abandonner après une ou deux années, ayant perdu tous leurs biens ${ }^{80}$. 
La crise et les faillites vont faire crever le ballon et corriger un peu ce que le journal appelle "the crazy for storekeeping"81. Les coupures de prix et la faiblesse des profits, que le journal attribue à l'encombrement du métier de détaillant, vont pouvoir cesser ${ }^{82}$. "Anything that tends to relieve the over-crowding which exists in the 'general store' line in Canada is welcome"83, écrit le Monetary Times en 1874; trois ans plus tard, il préconise toujours une réduction des effectifs de "l'armée commerciale" période, il y a un commerçant pour chaque soixante-quinze habitants, et un commis voyageur pour chaque vingt-deux commerçants.

La conjoncture économique aura donc joué un grand rôle dans la vie commerciale du pays en éliminant les plus faibles.

\section{CONCLUSION}

Cet examen du commerce de détail au début des années soixante-dix du XIXe siècle permet de relever certaines caractéristiques générales du petit commerce ou commerce de détail, mais aussi de voir l'analyse qu'en faisait un journal économique contemporain. Sans doute faudrait-il analyser les fondements de la pensée économique des rédacteurs du Monetary Times, qui semblent conservateurs sur le plan idéologique. Cependant, l'objet premier de ces quelques pages a plutôt été d'analyser le petit commerce.

L'histoire des réseaux de distribution est un champ encore peu exploré. Les années soixante-dix présentent une conjoncture qui met mieux en lumière la nature et l'organisation de ce secteur de la vie économique. À des années de grande prospérité (notamment entre 1869 et 1873) succède une période de grande crise (de 1874 à 1878).

De nombreux facteurs expliquent la prospérité d'avant 1873, la plupart étant externes au Canada. Par ailleurs, dans un nouveau pays comme le Canada, la demande de produits était généralement élevée. En même temps, les pays européens produisaient alors de grands surplus qu'ils voulaient vendre à travers le monde. Il existait donc de fortes pressions venant d'outre-Atlantique. Les facteurs qui sous-tendent l'expansion manufacturière de l'Europe sont bien connus en histoire économique: le développe-

\footnotetext{
$81 \quad M T, 25$ juillet $1879,124$.

$82 M T, 21$ décembre $1877,729-730$

$83 M T, 15$ octobre 1874,428 .

$84 M T, 21$ décembre $1877,729-730$.
} 
ment des transports d'abord, puis tout un train d'inventions et de techniques qui contribuent à l'abaissement des coûts de production. $\mathrm{Au}$ Canada, le développement d'un système de canaux durant les années quarante, puis la construction d'un premier réseau ferroviaire durant les années cinquante ont grandement facilité les échanges commerciaux. L'amélioration des moyens de transport contribue aussi à abaisser les coûts et favorise un peu partout la spécialisation, dont la suite naturelle est le développement d'une économie monétaire. Dans la mesure où la main-d'oeuvre exerce une activité plus ou moins spécialisée et touche par conséquent un salaire, elle doit acheter chez le marchand un nombre croissant de produits.

Les recensements du XIXe siècle ne permettent pas de délimiter avec précision cette classe comprenant des marchands généraux, des petits commerçants et des détaillants qui, à l'époque, se confondent généralement. Nous avons donc affaire à une catégorie socioéconomique mal définie, qui constitue néanmoins un réseau complexe assurant la distribution des produits à l'intérieur du pays, aussi entre le Canada et l'étranger.

Les marchands s'approvisionnent chez les grossistes, rarement au début du XIXe siècle, mais de plus en plus régulièrement après l'amélioration des transports. La surproduction en Europe encourage les marchands à commander trop pour leurs moyens et surtout à recourir abusivement au crédit. Les grossistes, les marchands de détail, les consommateurs, tous forment une immense pyramide de crédit, liés les uns aux autres par l'endettement. Pour accélérer les ventes, on a aussi de plus en plus recours aux commis voyageurs qui circulent à travers tout le pays pour recevoir les commandes des marchands.

Quand éclate la crise de 1873 , toutes les instances réclament leurs créances. La mévente de certains produits prive les consommateurs de liquidités, ce qui entraîne les marchands créanciers à la faillite et les empêche de remettre les sommes dues aux grossistes. Pour le journal Monetary Times, qui a déploré depuis des années les abus du crédit, la crise provoque néanmoins un effet salutaire en éliminant beaucoup de ces mauvais marchands qui ne connaissent pas leur métier, vivent au-dessus de leurs moyens, ne tiennent pas de livres et empruntent trop. Ce sont les plus inefficaces et les moins rentables qui partent, mais d'autres attendent en grand nombre pour les remplacer. 
En lisant le Monetary Times, on obtient certainement l'image d'un monde grouillant dans tout le secteur de la vente au détail. En général, les marchands semblent mal préparés, mal organisés et peu conscients des exigences de leur profession. Même en concédant le journalisme étant ce qu'il est - que le Monetary Times ait pu exagérer, on garde toujours l'impression que les marchands généraux constituent un groupe assez désordonné.

Pourtant, ils forment un chaînon indispensable à la distribution des marchandises à travers le pays. En les étudiant, on constate que les abus dénoncés par le Monetary Times tiennent non seulement au caractère des petits commerçants individuels, mais aussi aux intérêts de divers groupes qui s'en accommodent. L'ensemble du système de production et de distribution fonctionne de manière à faire parvenir les marchandises aux consommateurs, et le système de crédit sert au fonctionnement de l'ensemble. Ainsi, il répond autant aux besoins de la bourgeoisie productrice qu'à la demande des clients. 